The psychosocial and health effects of workplace reorganisation. 1. A systematic review of organisational-level interventions that aim to increase employee control

\author{
Matt Egan, Clare Bambra, Sian Thomas, Mark Petticrew, Margaret Whitehead, Hilary Thomson
}

J Epidemiol Community Health 2007;61:945-954. doi: 10.1136/jech.2006.054965

Objective: Systematic review of the health and psychosocial effects of increasing employee participation and control through workplace reorganisation, with reference to the "demandcontrol-support" model of workplace health.

Design: Systematic review (QUORUM) of experimental and quasi-experimental studies (any language) reporting health and psychosocial effects of such interventions.

Data sources: Electronic databases (medical, social science and economic), bibliographies and expert contacts.

Results: We identified 18 studies, 12 with control/comparison groups (no randomised controlled trials). Eight controlled and three uncontrolled studies found some evidence of health benefits (especially beneficial effects on mental health, including reduction in anxiety and depression) when employee control improved or (less consistently) demands decreased or support increased. Some effects may have been short term or influenced by concurrent interventions. Two studies of participatory interventions occurring alongside redundancies reported worsening employee health.

Conclusions: This systematic review identified evidence suggesting that some organisational-level participation interventions may benefit employee health, as predicted by the demand-control-support model, but may not protect employees from generally poor working conditions. More investigation of the relative impacts of different interventions, implementation and the distribution of effects across the socioeconomic spectrum is required.

See end of article for authors' affiliations

Correspondence to: Matt Egan, Medical Research Council Social and Public Health Sciences Unit, University of Glasgow, 4 Lilybank Gardens, Glasgow G128RZ, UK; matt@msoc.mrc.gla.ac.uk

Accepted 26 February 2007

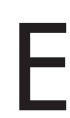
mployment is widely regarded as an impor- tant determinant of health. ${ }^{1}$ One of the most influential theories describing this relationship is the "demand-control-support" (DCS) model of workplace health, which hypothesises that employee health may be negatively associated with job demands and positively associated with control and social support in the workplace. ${ }^{1-6}$ Although the model continues to be debated, it has proved influential amongst policy-makers. The 2004 English public health strategy, Choosing health, recommends increasing job control as a key task for improving population health. ${ }^{7}$ We have conducted a systematic review of organisational-level workplace interventions that may achieve this key task.
Observational epidemiological studies have provided fairly consistent evidence that workplace control may be associated with health, but findings have been more mixed with regard to the full DCS model. ${ }^{8}$ Some commentators have emphasised the interactive effects of these psychosocial characteristics, suggesting that the combination of high demand and low control (and low support) adversely affects health, whilst the inverse of these combined characteristics enhances health. ${ }^{356}$ Some have prioritised specific parts of the model (e.g. suggesting that control may have stronger associations with health than demands). ${ }^{1}{ }^{10}$ In addition, researchers have highlighted potentially important individual factors not considered by the model (e.g. personal modes of coping and need to control), and alternative models such as "effortreward imbalance" have been advanced. ${ }^{11}$ The degree to which workplace psychosocial factors explain health outcomes independently of variables such as status in the community, income and health behaviours has also been questioned. ${ }^{12}$

Intervention evaluations have been advocated as a means of testing the validity and applicability of psychosocial theories. Such evaluations have been called "the bullet that psychosocial epidemiology has to bite" to provide evidence for this purpose and influence policy. ${ }^{13}$ Evaluations of interventions to improve workplace control may help us identify ways not only to improve employee health, but also to reduce health inequalities, as some evidence suggests a social gradient in exposure to work control (i.e. lower occupation groups may experience less control). ${ }^{14}$

\section{ORGANISATIONAL INTERVENTIONS}

Karasek (a theorist in this field) categorised workplace psychosocial interventions by distinguishing "organisational-level" interventions, aimed at changing the psychosocial environment, from "individual-level" interventions that focus on how individuals behave and cope with that environment. He argued that organisational interventions were preferable as preventative measures because they addressed the causes of unhealthy working environments. ${ }^{4}$

The systematic review presented here focuses on site-specific (rather than area-wide legal or socioeconomic transitions ${ }^{4}$ ) organisational interventions designed to increase employees' opportunities to make decisions or participate in decision-making processes at work. As managerial structures may change to facilitate employee 
participation and control, Karasek describes these as "macrolevel" interventions that cut across workplace hierarchies. In a companion systematic review we investigate the health effects of more localised "micro-level" organisational interventions that affect workers' daily task structures (C Bambra et al., unpublished data).

We know of no other systematic review that focuses on participation interventions. Existing reviews tend to broadly scope workplace intervention evaluations, to include studies that report relatively few data on either health or psychosocial outcomes or to focus on individual-level interventions. ${ }^{4}{ }^{15-21}$

Systematic reviews are increasingly advocated for identifying and synthesising evaluative evidence on the wider determinants of health and health inequalities. ${ }^{22}{ }^{23}$ Employment has been highlighted as a policy area in need of such reviews. ${ }^{23}$ In this systematic review we ask whether organisational-level interventions designed to increase employee participation/ control lead to health effects predicted by the DCS model.

\section{METHODS}

\section{Inclusion and exclusion}

We included experimental, prospective and retrospective studies evaluating organisational-level interventions (single or multiple interventions) intended to increase employees' opportunities to make decisions or participate in decision-making.

We included only studies that evaluated both the psychosocial and health effects of such interventions, so we could explore the relationship between these effects. Psychosocial outcomes included self-reported demand, control and support or related measures (e.g. work complexity, autonomy, satisfaction with colleagues). Health outcomes included self-reported physical health, mental health, absenteeism and physical measures. We excluded studies that focused exclusively on workplace injuries, or which did not report workplace psychosocial characteristics beyond general job satisfaction.

\section{Search strategy}

We searched for documents of any type, language or nationality. We developed a sensitive electronic search strategy using lists of terms associated with workplace reorganisation, psychosocial outcomes and health (see our protocol: http://www. msoc-mrc.gla.ac.uk/Evidence/Research/Research_MAIN.html), and searched databases from start date to November 2006 (see box 1). We also searched SIGLE, PAIS, Dissertation Abstracts and other Internet resources, manually searched bibliographies and contacted experts.

We initially located 65282 titles and abstracts, and retrieved 733 for detailed examination. All empirical studies of organisational-level employee participation interventions were independently assessed by two reviewers (CB and ME) for relevance and methodological quality (tables $1-4$ ).

\section{Data extraction, appraisal and synthesis}

Critical appraisal criteria were adapted from the systematic review methodological literature and existing systematic reviews of complex interventions. ${ }^{24-27}$ Data were abstracted by one reviewer (ST) and checked by another (ME). We calculated effect sizes and 95\% confidence intervals (CIs) where possible, but it should be noted that these sometimes differed from $p$ values reported in the original articles, possibly because our calculations relied on summarised final sample size data reported in journals, rather than original datasets. Heterogeneity in interventions, study designs, comparison groups, outcome measures and reporting of data made metaanalysis and comparisons of effect sizes between studies problematic. Therefore, we used narrative synthesis: categorising and tabulating data by intervention type, methodology, setting and outcome, and describing studies in a narrative that
Box 1 Electronic databases searched (hosts given in parentheses)
ASSIA (CSA)

British Library catalogue

Business Periodicals Premier

Conference Papers Index (CSA)

COPAC

Econlit (Dialog/Ovid)

Electronic Collections Online (OCLC

firstsearch)

Embase (Dialog)
ERIC (CSA/Dialog)

Index to theses

Medline (Ovid/Dialog)

NTIS (free version)

Psycinfo (Dialog/Ovid)

Social Sciences Citation Index

(MIMAS)

Sociological abstracts (CSA)

Zetoc emphasised more methodologically robust (e.g. prospective, controlled) studies. ${ }^{242528} 29$

\section{RESULTS}

We identified 18 studies (published between 1981 and 2006) that examined both the health and psychosocial impacts of organisational interventions aimed at increasing employee participation/control. ${ }^{30-48}$ Four were identified through manual searches, ${ }^{30} 364042$ the rest electronically.

Most included studies evaluated interventions involving "participatory" or "problem-solving" committees of employee representatives. These were usually established to identify ways of tackling workplace stressors, although one had wider powers concerning budgeting and human resources. ${ }^{32}$ Some participatory interventions were implemented in combination with individual-level interventions, ${ }^{38-41}$ ergonomic improvements, ${ }^{42}{ }^{43}$ or organisational downsizing (tables $1-4) .{ }^{44-48}$

We identified 12 prospective studies with non-randomised comparison groups..$^{30-42} 44$ Comparison groups typically consisted of employees from similar departments or workplaces to the intervention groups. Key findings from these prospective controlled studies are summarised in the text. We also identified three uncontrolled prospective $e^{37} 46$ and three retrospective $^{43478}$ studies (one of which was qualitative ${ }^{48}$ ). Tables 14 summarise findings and methodological details from all the studies.

\section{Single-intervention studies}

Six of the seven studies evaluating single participatory interventions were prospective with comparison groups. ${ }^{30-36}$ (table 1). Apart from one study of employees given more control over their working hours, ${ }^{36}$ the interventions took the form of employee committees to identify workplace stressors and ways to reduce them. One committee's role was semimanagerial. $^{32}$

Two cohort studies with comparison groups had civil service settings. One examined the effects of establishing problemsolving committees comprising managers, elected employee representatives and an external consultant at two regional public health departments in the USA. ${ }^{30}$ After 12 months, neither employees' adjusted mean depression scores nor rates of self-reported sleeping problems had changed significantly. There was little change $(\mathrm{p}>0.05)$ in self-reported demand, control or support (Job Contents Questionnaire (JCQ)).

The other examined a UK central government office where a workers' committee of volunteer employee representatives, moderated by a consultant psychologist, was established. ${ }^{31}$ After 12 months, mean scores for "sense of control" increased in the intervention group from 10.31 (95\% CI 9.65 to 10.97 ) to 12.70 (95\% CI 11.96 to 13.44$)(\mathrm{p}<0.0001)$ and decreased in the comparison group from 10.86 (95\% CI 10.16 to 11.56 ) to 10.65 (95\% CI 9.40 to 11.90 ). Mean Occupational Stress Indicator scores for mental ill health improved from 57.56 (95\% CI 54.19 


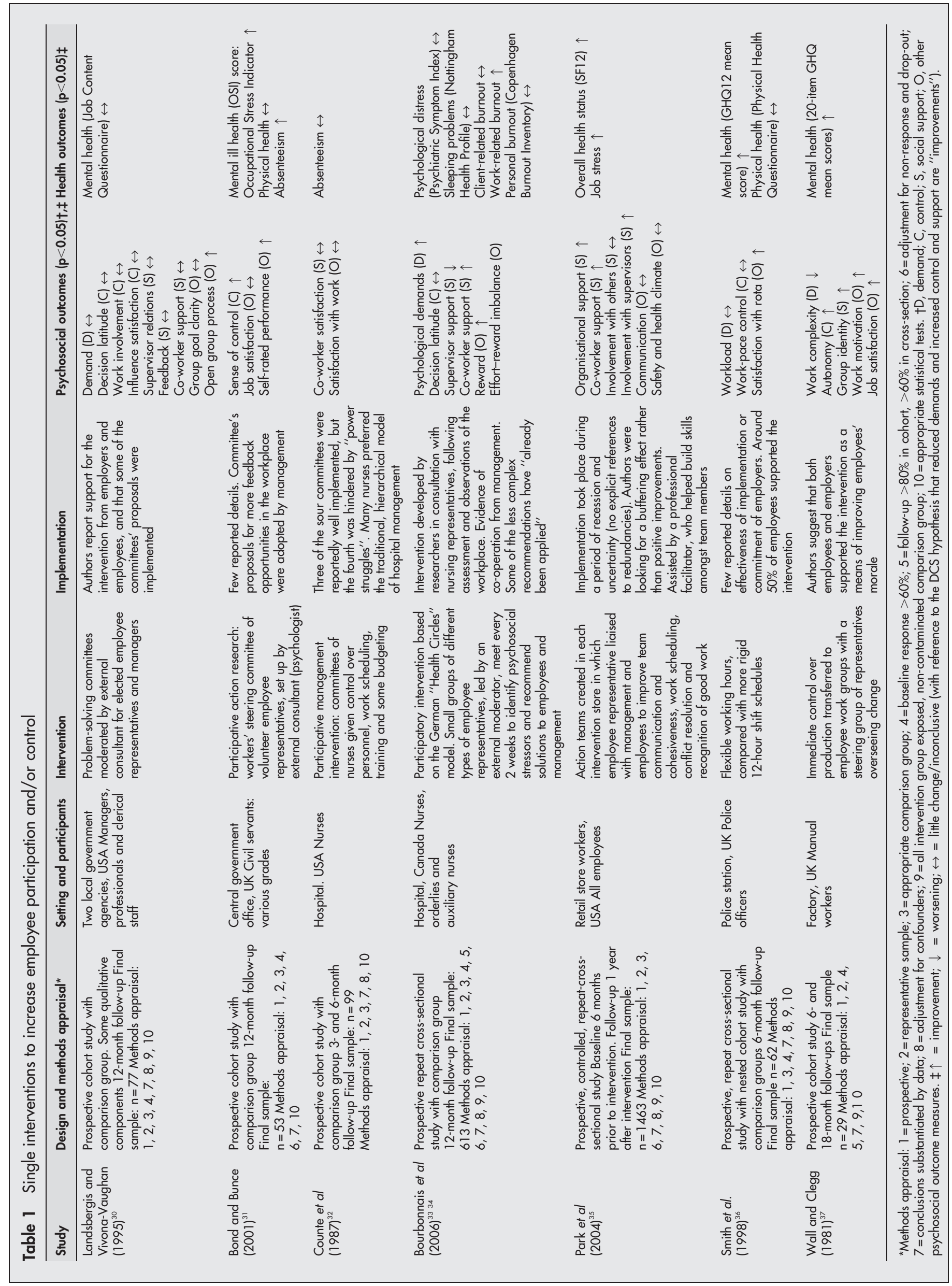




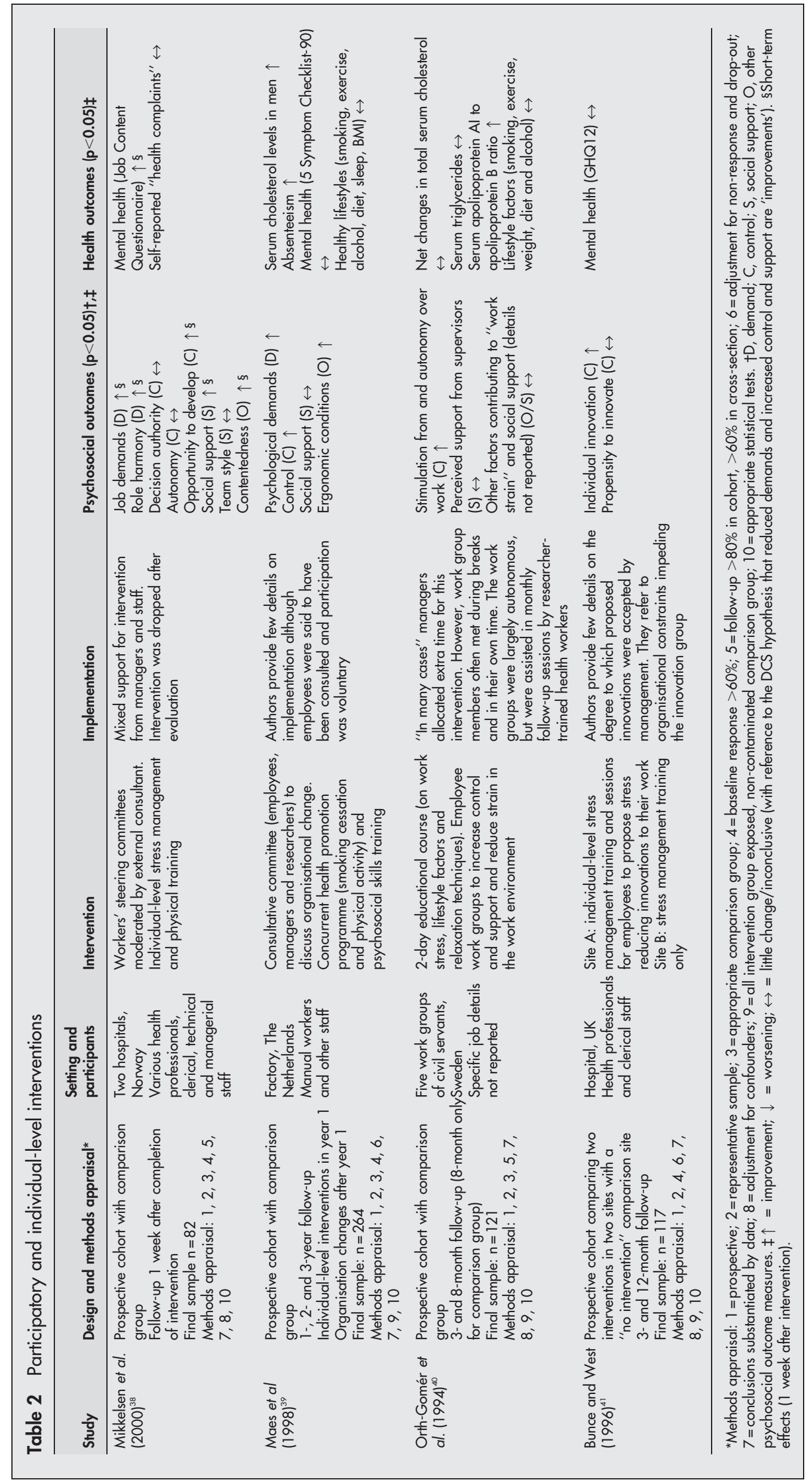




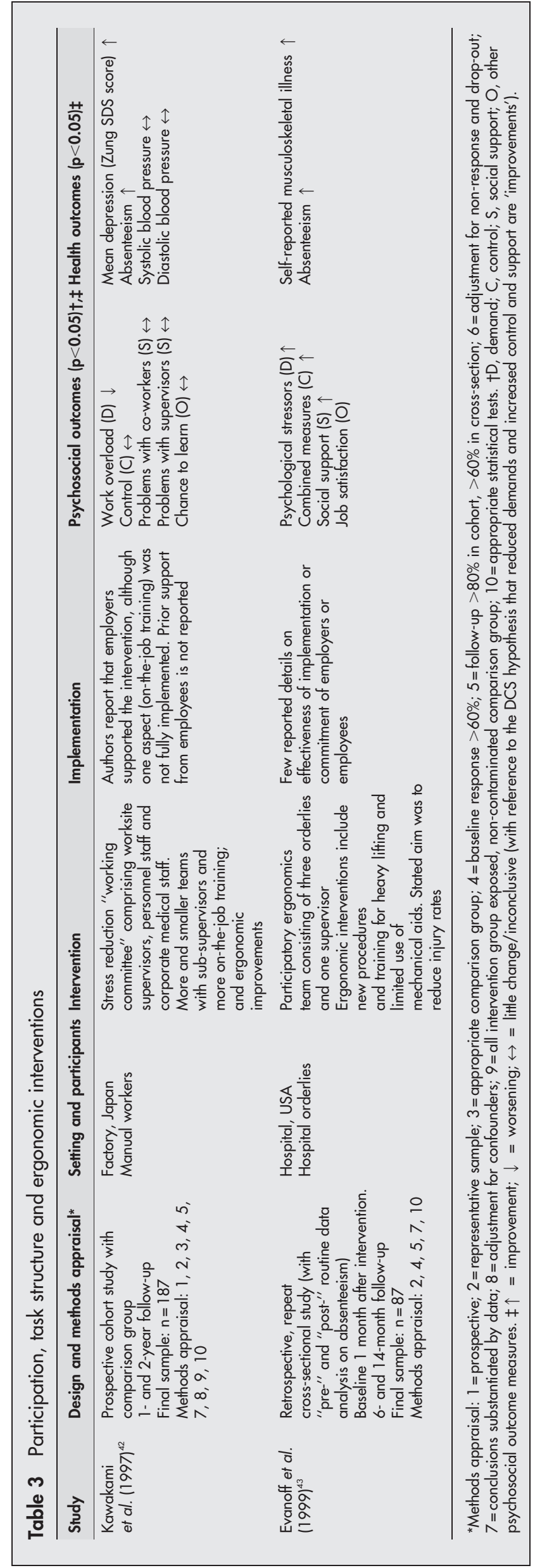

to 60.93 ) to 52.27 (95\% CI 45.96 to 58.58 ) in the intervention group relative to the comparison group's increase from 53.19 (95\% CI 49.45 to 56.93 ) to 58.96 (95\% CI 53.99 to 63.93 ) $(\mathrm{p}=0.014)$. The intervention group also experienced reductions in routinely recorded sickness absence.

A prospective cohort study found that a participative management intervention in a hospital in the USA appeared to have little effect $(p>0.05)$ on psychosocial and health outcomes. $^{32}$

A Canadian repeat cross-sectional study evaluated a "quality circles" hospital intervention involving externally moderated, problem-solving committees of employee representatives focusing on workplace stressors. After 12 months, mean JCQ scores decreased for "psychological demands" (mean of differences between before and after scores: experimental group $=-0.56$ (95\% CI -0.94 to -0.18$)$; comparison group $=-0.31$ (95\% CI -0.68 to 0.07 ); $\mathrm{p}=0.015$ ); and "supervisor support" (mean differences: experimental group $=-0.57$ (95\% CI -0.86 to $-0.27)$; comparison group $=-0.92(95 \% \mathrm{CI}-1.21$ to -0.63$)$; $\mathrm{p}=0.028) .{ }^{33}{ }^{34}$ Improvements were reported for "CO-worker support" (mean differences: experimental group $=0.04(95 \%$ CI -0.16 to 0.25$)$; comparison group $=-0.12$ (95\% CI -0.32 to $0.08) ; \mathrm{p}=0.056)$, "reward" (mean differences: experimental group $=0.41 \quad(95 \% \quad$ CI $\quad-0.01 \quad$ to 0.83$) ; \quad$ comparison group $=-0.16(95 \%$ CI -0.58 to 0.25$) ; p=0.001)$ and "effort-reward imbalance" (mean differences: experimental group $=-0.04 \quad(95 \%$ CI -0.07 , to -0.01$) ;$ comparison group $=-0.01(95 \%$ CI -0.04 to 0.01$) ; p=0.002)$, but not for "decision latitude" $(\mathrm{p}=0.382)$. Psychiatric Symptom Index mean scores for "psychological distress" $(p=0.205)$ and selfreported sleeping problems $(\mathrm{p}=0.210)$ were inconclusive, as were Copenhagen Burnout Inventory mean scores $(\mathrm{p}>0.8)$, except for a reduction in "work-related burnout" (mean differences: experimental group $=-1.83(95 \%$ CI -3.58 to $-0.09)$; comparison group $=0.06(95 \%$ CI -1.66 to 1.78$)$; $\mathrm{p}=0.034)$.

A US repeat cross-sectional study evaluated the effects of externally moderated "problem-solving" committees of employee representatives in 11 retail stores. ${ }^{35}$ Relative to employees in 10 comparison stores, the intervention group reported improvements in mean scores (Eisenberger and Worksite Health Climate Scales) for "organisational support" $(p=0.001)$, "co-worker support" $(p<0.001)$, "involvement with supervisors" $(p=0.02)$, "overall health status" (SF12) $(p=0.004)$ and "job stress" (Cohen's six-item scale) $(p=0.02)$ after 12 months. Inconclusive evidence of improvements were found for "involvement with others" $(p=0.06)$, "communication" $(p=0.07)$ and "safety and health climate" $(p=0.07)$.

A prospective, repeat cross-sectional study with nested cohort study of two UK police departments found that GHQ12 mean scores (psychological well-being) improved after 6 months for workers given more control over shift rotas compared with employees with fixed rotas $(\mathrm{p}<0.05) .{ }^{36}$ Changes in self-reported demand, control and physical health varied little between the two groups.

\section{Multi-intervention studies}

Eleven (including six controlled) studies examined participation interventions delivered as part of packages of interventions. $^{38-48}$

\section{Participation and individual-level interventions}

Four studies evaluated employee committees combined with individual-level health promotion, education and behaviour interventions, such as anti-smoking or physical activity interventions, and training in relaxation techniques, stress reduction and communication skills (table 2)..$^{38-41}$ 


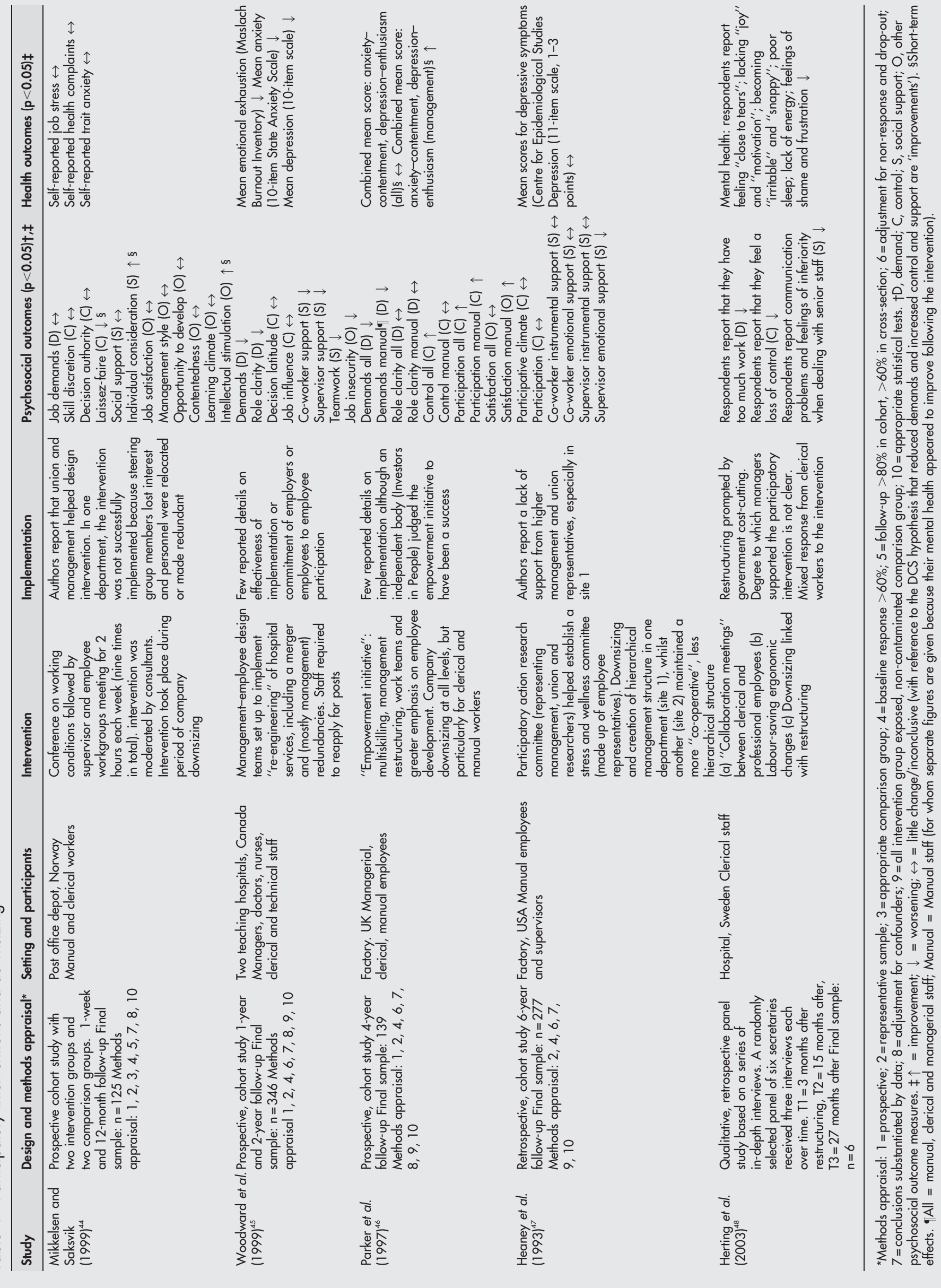


A prospective Norwegian hospital study ${ }^{38}$ examined the impact of stress management and physical training sessions combined with an externally moderated workers' steering committee to improve health and organisational performance. After adjusting for demographic characteristics, mean JCQ scores were found to have decreased after a week for "job demands" (from 13.99 to 13.77, relative to the comparison group (in which the JCQ score increased from 12.29 to 14.86): ANCOVA, $\mathrm{p}<0.05$ ). "Opportunity to develop" improved (from 32.34 to 32.68 ) relative to the comparison group (36.50 to 32.58) ( $<<0.05)$, as did mean Work Apgar Questionnaire scores for "social support" (from 18.43 to 19.84) relative to the comparison group (20.64 to 19.21) $(\mathrm{p}<0.05)$ and "role harmony" (3.86 to 4.43 ) relative to the comparison group (4.88 to 3.93) (p<0.05). "Work-related stress" (JCQ) fell from 6.55 to 5.95 relative to the comparison group (4.07 to 7.36 ) $(\mathrm{p}<0.05)$.

A similar package of interventions (plus smoking restrictions) was evaluated in a Dutch prospective cohort study. ${ }^{39}$ Individual-level interventions were implemented in a factory between baseline (T1) and follow-up at 12 months (T2). Organisational interventions occurred between T2 and followup at 24 (T3) and 36 (T4) months. Mean scores for "control" (Work Stress Questionnaire) increased significantly in the intervention group (from 2.34 (95\% CI 2.27 to 2.41 ) to 2.53 (95\% CI 2.48 to 2.58$)$ ) but not in the comparison group (from $2.50(95 \%$ CI 2.44 to 2.56$)$ to $2.54(95 \%$ CI 2.48 to 2.60$)$ ) between T2 and T3 $(p<0.01)$. Mean scores for "psychological demands" changed little in the intervention group (from 1.49 (95\% CI 1.43 to 1.55 ) to 1.53 (95\% CI 1.46 to 1.60$)$ ) but increased significantly in the comparison group (from 1.49 (95\% CI 1.43 to 1.55$)$ to 1.64 (95\% CI 1.57 to 1.71$)$ ) between T3 and T4 $(\mathrm{p}<0.01)$. Serum cholesterol levels improved in men between T1 and T2 (men: $\mathrm{p}=0.02$; women: $\mathrm{p}=0.09$ ).

Four groups of civil servants in Sweden participated in a 2day course on dealing with stress, healthy lifestyles and relaxation techniques. ${ }^{40}$ Over the following 8 months, employee workgroups met to identify and recommend solutions to workplace stressors. A prospective cohort study with comparison group found that stimulation from and autonomy over work improved significantly in the intervention group $(\mathrm{p}<0.01)$ but remained unchanged in the comparison group. There was inconclusive evidence of an increase in perceived support from supervisors $(p<0.1)$. A $6 \%$ mean reduction in the ratio between apolipoproteins B and AI (which may indicate reduced cardiovascular risk) was reported $(p<0.05)$, without any concomitant change in the control group. Little evidence of effect was found for measures of total serum cholesterol, serum triglycerides and lifestyle factors (smoking, exercise, weight, diet and alcohol consumption).

A prospective cohort study compared an individual-level intervention, a combined individual and organisational intervention, and a no-intervention control in three UK hospital departments. ${ }^{41}$ Employees who received combined interventions reported improved "individual innovation" at both 3 and 12 months' follow-up (z-scores $=-0.17$ (T1), 0.01 (T2), and $0.92(\mathrm{~T} 3))$ compared with employees receiving no intervention $(\mathrm{z}$-scores $=0.17(\mathrm{~T} 1),-0.02(\mathrm{~T} 2)$ and $0.09(\mathrm{~T} 3)(\mathrm{p}<0.001)$. The authors reported little comparative change in job-induced tension or psychological strain (GHQ12) amongst employees receiving the combined intervention.

\section{Participation and ergonomic interventions}

One controlled ${ }^{42}$ and one uncontrolled study ${ }^{43}$ evaluated participatory committees combined with ergonomic interventions, i.e. attempts to reduce physical discomfort and workplace injuries by modifying physical environments (including technological improvements) and advising on posture and lifting (table 3).

In a Japanese factory, a committee of worksite supervisors, medical staff and personnel met over several months to devise a programme to reduce worksite stressors identified by the supervisors. This involved increased teamworking, overtime and ergonomic improvements. ${ }^{42}$ After 2 years, a prospective controlled study found no significant psychosocial changes except for an increase in "work overload" (from 26\% to 43\%) relative to the comparison group ( $28 \%$ to $26 \%)(p=0.054)$. The authors also reported reductions in short-term ( $1-5$ days) absenteeism in the intervention group (52\% to $34 \%$ ) relative to the comparison group ( $33 \%$ to $37 \%)(p=0.034)$; and mean Zung self-rated depression scores (intervention group 41.1 to 38.6; comparison group 41.5 to $42.3 ; \mathrm{p}=0.025$ ) amongst men.

\section{Negative organisational change}

Amongst five studies evaluating employee participation interventions intended to reduce the negative effects of organisational downsizing (e.g. job insecurity/redundancies), only one was prospective and controlled (table 4). ${ }^{45}$ This study in a Norwegian post office compared departments that instigated externally moderated working conditions groups (involving supervisors and employees) with a no-intervention group of employees. The authors reported little difference in psychosocial or health outcomes after 1 week, and none after 12 months, except for self-reported "commitment" $(p<0.05)$.

\section{Health inequalities}

Studies reporting differential effects by social group could potentially shed light on how paticipation interventions might help tackle health inequalities. Only one included study reported a differential effect of an intervention by gender, finding that serum cholesterol levels improved for men but not women (T1-T2: men, $\mathrm{p}=0.02$; women, $\mathrm{p}=0.09) .{ }^{39}$ One controlled study reported that participation interventions preceded psychosocial improvements $(p<0.05)$ for black and Hispanic, but not white, employees, but similar interactions were not observed for overall health status and job stress. $^{35}$

Several studies looked at particular occupational groupsmanual workers, clerical staff, health professionals, police or managers-and found health improvements following some of the interventions reviewed here. Only one (uncontrolled) study compared an intervention's effects across two or more occupational groups. It found improvements in mean scores for strain (anxiety-contentment and depression-enthusiasm 5-point scales) for manual factory workers (from 2.71 to 2.45; $\mathrm{p}<0.01$ ), but not managers or clerical staff $(\mathrm{p}>0.05)$, 4 years after a participation intervention implemented during company downsizing. ${ }^{46}$

\section{Psychosocial factors and health}

Eight (including four controlled) studies reported post-intervention improvements in measures of control/participation. ${ }^{31}{ }^{37-}$ 414346 Seven of these also reported health improvements, whilst one uncontrolled study reported little change in health. ${ }^{41}$ Two studies of participation interventions during downsizing reported declines in employee control: one also reported worsening health, ${ }^{48}$ whilst the other reported no significant health effects. ${ }^{44}$

Reductions in demands were reported in three controlled studies $^{33} 343839$ and an uncontrolled study ${ }^{43}$ and at least one health outcome improved each time.

Two controlled ${ }^{35}$ and two uncontrolled studies ${ }^{37}{ }^{43}$ reported improvements in both support and health. One controlled study found little change in health despite improvements in support ${ }^{44}$ and another found limited health improvements when support 
from colleagues improved but supervisor support worsened..$^{33} 34$ Reduced support was reported along with worsening health in two uncontrolled studies ${ }^{45}$ and little health impact was observed in another. ${ }^{47}$

Regarding the hypothesis that decreased demands and increased control and support interact to enhance health, ${ }^{3} 56$ this combination was reported in only two studies reviewed here (one of which was uncontrolled, while the other followed up outcomes for only l week), whilst a third study reported decreased demands, increased control and little change in support. $^{383943}$ All three reported improvements in at least one health outcome. This evidence broadly fits the above hypothesis but is insufficient to validate it.

The corollary of the above hypothesis is suggested by a qualitative study reporting that increased demands and decreased control and support occurred with worsening health indicators. ${ }^{48}$ However, one controlled study reported health improvements despite increased demands and little change to control or support. ${ }^{42}$ In two uncontrolled studies, health improved alongside increased demands and improved control $^{3746}$ and support. ${ }^{37}$

\section{DISCUSSION}

\section{Evidence quality and availability}

This systematic review identified 18 studies that evaluated both the health and psychosocial impacts of organisational-level interventions intended to increase employee participation in workplace decision-making. None of the 12 controlled studies found evidence of health deterioration, whilst eight ${ }^{31}$ 33-36 38-40 42 (along with three uncontrolled studies ${ }^{37} 43$ 46 ) found evidence of health improvements.

Some of the reported health measures might more properly be considered proxies (e.g. some "burnout" measures and biomarkers). Most measures were self-reported and the length of follow-up ranged from 1 week to 4 years. Adjustments for confounding were often poorly reported, absent or limited to demographic rather than health variables. Evaluations of multiple interventions tended not to identify effects specific to the organisational-level interventions most relevant to this review.

However, evaluations of the health effects of complex social interventions are relatively rare (e.g. compared with individuallevel and therapeutic interventions) and often take the form of "natural experiments" requiring pragmatic methodological designs. Although we identified no randomised controlled trials, the number of prospective studies with comparison groups in this review compares favourably with the evidence available for many other types of sociostructural interventions affecting health ${ }^{26} 49-51$

\section{Research and policy}

More robust evidence is required, but the findings from this review remain broadly compatible with the UK Department of Health's view that increasing employee control is a key task for policy-makers. ${ }^{7}$ We found that health improvements (e.g. improved mental health and reduced sickness absenteeism) may sometimes result from such interventions. The only negative health effects were reported in two uncontrolled studies that may have been confounded by organisational downsizing. ${ }^{45}{ }^{48}$ Qualitative evidence suggests that job insecurity and communication barriers associated with workplace hierarchies may hinder participation interventions. ${ }^{48}$

What little evidence is available suggests that participation interventions may benefit lower grade workers and employees belonging to ethnic minorities. Hence, the potential of such interventions for reducing workplace health inequalities is worthy of further investigation.

\section{Demand, control and support}

To establish whether health outcomes are conditional on psychosocial improvements resulting from the interventions, we suggest that future prospective studies should distinguish between employees (from both the intervention and control groups) who do and do not experience psychosocial improvements in demand, control or support. If an intervention influences health through a psychosocial pathway, greater health improvements would be expected amongst intervention group participants who report psychosocial improvements in the work environment (compared with other participants).

The evidence we identified does not report data in this way, but our findings do broadly fit health outcomes hypothesised by the DCS model. Interventions that improved workplace control and/or support did tend to improve employee health. Health improvements did not occur when control or support worsened, except in one case where limited health improvements occurred when colleagues' support improved but supervisor support worsened..$^{34}$ Interventions that reduced demands also improved health (as hypothesised by the model). However, sometimes health improved even when the intervention appeared to increase demands.

We identified limited and somewhat inconsistent evidence to support the hypothesis that low demands and high control (and support) combine to enhance health. The evidence also sheds little light on how other psychosocial models may compare with the DCS model (one study reported improvements in work burnout, improved effort-reward model measures and mixed effects on DCS measures ${ }^{33}{ }^{34}$ ).

In an accompanying review of "micro-level" organisational interventions affecting employees' task structures, we also found the DCS model to be a useful (but not infallible) predictor of health outcomes (C Bambra et al., unpublished data). However, these "micro-level" interventions tended to increase demand, decrease control and negatively affect health. In contrast, the participation interventions reviewed here usually had benign or beneficial, but not adverse, health effects (unless accompanied by redundancies).

\section{LIMITATIONS}

There are several limitations to the interpretation of the studies reviewed here. First, in many of the original papers the reporting of the interventions was generally poor or difficult to assess, even with the help of implementation evaluation tools. ${ }^{27}$ There is a lack of evidence that the interventions were actually implemented in full, or at all. In the tables we have tried to summarise reported details of intervention implementation, but many papers offered few clues about this. These issues will form the basis of a future paper on implementation. Obviously, it strikes at the core of evaluation practice - what are the authors measuring if the intervention was little more than a paper exercise?

Second, several of the organisational interventions aimed at changing the psychosocial environment took place alongside individual-level health education or ergonomic initiatives. It is generally not possible to separate out the health effects of the different types of intervention, though some reported health outcomes, such as reductions in injuries and changes in lifestyles such as smoking and diet, could plausibly be attributed to the intervention more directly aimed at these outcomes.

Third, five of the interventions are reported to have taken place while companies were undergoing downsizing and redundancies. In such cases, an absence of negative health effects may indicate a protective effect from increased participation, counteracting the negative psychosocial impacts of downsizing. A controlled study is required to test this 


\section{What is already known on this subject}

The demand-control-support model of psychosocial workplace health has been particularly influential amongst researchers and policy-makers interested in employee health and health inequalities.

It posits that health is positively associated with employees' sense of control and social support and negatively associated with workplace demands, suggesting that interventions that modify these work characteristics appropriately may benefit employee health.

\section{What this study adds}

First systematic review of the health effects of interventions aimed at improving employee control and/or participation in workplace decision-making.

Interventions that successfully improve employees' sense of control are potentially health improving, although they may not protect workers from generally poor working conditions.

\section{Policy implications}

We recommend that policies and interventions that aim to increase job control and autonomy amongst employees should be incorporated in public health strategies.

hypothesis. The only one we identified found no evidence of health protection. ${ }^{44}$ Perhaps it is unrealistic to expect the relatively modest interventions of establishing participatory committees to protect workers from substantial deteriorations in workplace conditions. Nor are they necessarily responsible for observed health improvements amongst employees who believe they have survived a downsizing period. ${ }^{46}$

The hypotheses and methodological issues discussed here should be taken into account, both in the interpretation of existing studies and in the design of future intervention evaluations.

\section{CONCLUSION}

This systematic review of "participatory" interventions has identified evidence of some health benefits occurring when employee control improved or (less consistently) demands decreased or support increased. This finding fits with evidence from some observational epidemiological studies ${ }^{18}$ and is compatible with policy directives on participation at work. ${ }^{752}$ More robust prospective studies along the lines described above, with improved reporting of intervention implementation and differential impacts for different socioeconomic groups, are required to provide a stronger evidence base. The evidence we did identify suggests that the strategy of reorganising workplaces to facilitate employee participation and control offers a potential means of improving employee health and well-being, although the most effective means of implementing this strategy needs to be better understood.

\section{CONTRIBUTORS}

ME planned the study, collected and analysed data and is lead author and guarantor. $\mathrm{CB}, \mathrm{MP}, \mathrm{ST}, \mathrm{MW}$ and $\mathrm{HT}$ assisted in all aspects of the study including writing-up.

\section{ACKNOWLEDGEMENTS}

We thank N Barry, S Macintyre, CRD and the peer reviewers. Research funded as part of the ESRC Centre for Evidence-based Public Health Policy (ESRC grant H141251011), and MP is supported by the Chief Scientist Office of the Scottish Executive. The funders played no part in the conduct or reporting of the research.

\section{Authors' affiliations}

Matt Egan, Sian Thomas, Mark Petticrew, Hilary Thomson, Medical Research Council Social and Public Health Sciences Unit, University of Glasgow, UK

Clare Bambra, Centre for Public Policy and Health, School for Health, University of Durham, UK

Margaret Whitehead, Division of Public Health, University of Liverpool, UK

Sources of support: Economic and Social Research Council and the Chief Scientist Office of the Scottish Executive Health Department.

Competing interests: None.

Ethics approval: Ethics approval not required (literature review).

\section{REFERENCES}

1 Marmot M, Siegrist J, Theorell T. Health and the psychosocial environment at work. In: Marmot M, Wilkinson R, eds. Social determinants of Health. Oxford: Oxford University Press, 1999

2 Karasek R, Baker D, Marxer F, et al. Job decision latitude, job demands and cardiovascular disease: a prospective study of Swedish men. Am J Public Health $1981 ; 71: 694-795$

3 Karasek R, Theorell T. Healthy work: stress, productivity and the reconstruction of working life. New York: Basic Books, 1990.

4 Karasek R. Stress prevention through work reorganisation: a summary of 19 case studies. Conditions of Work Digest 1992;11:23-42.

5 van Vegchel N, de Jonge J, Lansbergis PA. Occupational stress in (inter)action: the interplay between job demands and job resources. J Organizational Behav 2005;26:535-60.

6 Johnson J, Hall E. Job strain, work place social support, and cardiovascular disease: a cross-sectional study of a random sample of the Swedish working population. Am J Public Health 1988;78:1336-42.

7 Department of Health. Choosing health: Making health choices easier. London: HMSO, 2004

8 Marmot M, Theorell T, Siegrist J. Work and coronary heart disease. In: Stansfeld S, Marmot M, eds. Stress and the heart. Psychosocial pathways to coronary heart disease. London: BMJ Books, 2002:50-71

9 Siegrist J, Marmot M. Health inequalities and the psychosocial environment - two scientific challenges. Soc Sci Med 2004;58:1463-73.

10 Godin I, Kittel F. Can we disentangle life course processes of accumulation, critical period and social mobility? An analysis of disadvantaged socio-economic positions and myocardial infarction in the Stockholm Heart Epidemiology Program (SHEEP). Soc Sci Med 2003;58:1543-53.

11 Siegrist J. Adverse health effects of high effort/low-reward conditions. J Occup Health Psychol 1996;1:27-41.

12 Bartley M. Health inequality: an introduction to theories, concepts and methods. Cambridge: Polity Press, 2004

13 Macleod J, Davey Smith G. Psychosocial factors and public health: authors' reply. J Epidemiol Community Health 2003;57:553-6.

14 Marmot M, Bosma H, Hemingway $\mathrm{H}$, et al. Contribution of job control and other risk factors to social variations in coronary heart disease incidence. Lancet 1997;350:235-9.

15 Edwards DJ. A systematic review of stress and stress management interventions for mental health nurses. J Adv Nurs 2003;42:169-200.

16 Michie S, Williams S. Reducing work related psychological ill health and sickness absence: a systematic literature review. Occup Environ Med 2003:60:3-9.

17 van der Klink JJL, Blonk R, et al. The benefits of interventions for work-related stress. Am J Public Health 2001;91:270-6.

18 Platt S, Pavis S, Akram G. Changing labour market conditions and health: a systematic literature review (1993-98). Dublin: European Foundation for the Improvement of Living and Working Conditions, 1999.

19 Van der Hek H, Plomp HN. Occupational stress management programmes: a practical overview of published effect studies. Occup Med 2001;47(3):133-41

20 Landsbergis $\mathbf{P}$, Cahill J, Schnall $P$. The impact of lean production and related new systems of work organisation on worker health. J Occup Health Psychol 1999;4:108-30.

21 Parkes KR, Sparkes TI. Organizational interventions to reduce work stress: are they effective? HSE Contract Research Report 193/1998. Colegate, UK: HMSO, 1998.

22 Wanless D. Securing good health for the whole population: Final report. London: HM Treasury, 2004

23 Mackenbach JP, Bakker BB and the EU Network on Interventions and Policies to Reduce Inequalities in Health. Tackling socioeconomic inequalities in health: analysis of European experiences. Lancet 2003;362:1409-14.

24 NHS Centre for Reviews and Dissemination. Undertaking systematic reviews of research on effectiveness: CRD's guidance for those carrying out or commissioning reviews. York: NHS CRD, 2001. 
25 Clarke M, Oxman AD, eds. Cochrane reviewers' handbook. Oxford: Update Software, 2004.

26 Ogilvie D, et al. Systematic reviews of health effects of social interventions. 2. Best available evidence: how low should you go? J Epidemiol Community Health 2005;59:886-92.

27 Rychetnik L, Frommer M, Hawe P, et al. Criteria for evaluating evidence on public health interventions. J Epidemiol Community Health 2002;56:119-127.

28 Boaz A, Ashby D, Denyer D, et al. A multitude of syntheses: a comparison of five approaches from diverse policy fields. Evidence and Policy 2006;2:479-502.

29 Petticrew M, Roberts H. Systematic reviews in the social sciences: a practical guide. Oxford: Blackwell Publishing, 2006.

30 Landsbergis $\mathbf{P}$, Vivona-Vaughan E. Evaluation of an occupational stress intervention in a public agency. J Organisational Behav 1995;16:29-48.

31 Bond FW, Bunce D. Job control mediates change in a work reorganization intervention for stress reduction. J Occup Health Psychol 2001;6:290-302.

32 Counte MA, Barhyte DY, Christman LP. Participative management among staff nurses. Hospital \& Health Services Administration 1987;32(1):97-108.

33 Bourbonnais R, Brisson C, Vinet A, et al. Effectiveness of a participative intervention on psychosocial work factors to prevent mental health problems in a hospital setting. Occup Environ Med 2006;63:335-42.

34 Bourbonnais R, Brisson C, Vinet A, et al. Development and implementation of a participative intervention to improve the psychosocial work environment and mental health in an acute care hospital. Occup Environ Med 2006;63:326-34.

35 Park KO, Schaffer BS, Griffin-Blake CS, et al. Effectiveness of a healthy work organization intervention: ethnic group differences. J Occup Environ Med 2004;46:623-34.

36 Smith L, Hammond T, Macdonald I, et al. 12-h shifts are popular but are they a solution? Int J Indust Ergon 1998;21:323-31.

37 Wall TD, Clegg CW. A longitudinal field study of group work redesign. J Occup Behav 1981;2:31-49.

38 Mikkelsen A, Saksvik PØ, Landsbergis P. The impact of a participatory organizational intervention on job stress in community health care institutions. Work \& Stress 2000;14:156-70.
39 Maes S, Verhoeven C, Kittel F, et al. Effects of a Dutch work-site wellness-health program: the Brabantia project. Am J Public Health 1998;88:1037-41.

40 Orth-Gomer K, Eriksson I, Moser V, et al. Lipid lowering through work stress reduction. Int J Behav Med 1994;1:204-13.

41 Bunce D, West MA. Stress management and innovation interventions at work. Human Relations 1996:49:209-32.

42 Kawakami M, Araki S. Effects of work-related symptoms among Japanese bluecollar workers. Scand J Work Environ Health 1997;23(1):54-9.

43 Evanoff BA, Bohr PC, Wolf $L D$. Effects of a participatory ergonomics team among hospital orderlies. Am J Indust Med 1999:35:358-65.

44 Mikkelsen A, Saksvik PO. Impact of a participatory organizational intervention on job characteristics and job stress. Int J Health Services 1999;29:871-93.

45 Woodward CA, Shannon HS, Cunningham C, et al. The impact of reengineering and other cost reduction strategies on the staff of a large teaching hospital - a longitudinal study. Medical Care 1999;37:556-69.

46 Parker SK, Chmiel N, Wall TD. Work characteristics and employee well-being within a context of strategic downsizing. J Occup Health Psychol 1997;2:289-303.

47 Heaney CA, Israel BA, Schurman SJ, et al. Industrial-relations, worksite stress reduction, and employee well-being - a participatory action research investigation. J Organizational Behav 1993;14:495-510.

48 Hertting A, Nilsson K, Theorell T, et al. Personnel reductions and structural changes in health care: work-life experiences of medical secretaries. J Psychosomat Res 2003:54:161-70.

49 Bambra C. Whitehead M, Hamilton V. Does "welfare to work" work? A systematic review of the effectiveness of the UK's welfare to work programmes for people with a chronic illness or disability. Soc Sci Med 2005;60:1905-18.

50 Egan M, Petticrew M, Ogilvie D, et al. New roads and human health: a systematic review. Am J Public Health 2003;93:1463-71.

51 Thomson $\mathrm{H}$, Petticrew $M$, Morrison $D$. Health effects of housing improvement: systematic review of intervention studies. BMJ 2001;323:187-90.

52 European Agency for Safety and Health at Work, EU Council Directive 89/391, 1989. http://europe.osha.eu.int/data/legislation/ (accessed 21 October 2005). 